Journal of Applied Pharmaceutical Science Vol. 6 (06), pp. 091-095, June, 2016

Available online at http://www.japsonline.com

DOI: $10.7324 /$ JAPS.2016.60616

ISSN 2231-3354 (cc) EY-NC-SA

\title{
Efficacy and Safety of Metformin in Weight Loss in Bahraini Population
}

\author{
Sayed Mahmood Al-qallaf \\ Pharmacy Program, College of Health Sciences, University of Bahrain, P.O. Box 32038, Kingdom of Bahrain.
}

\begin{tabular}{|c|c|}
\hline ARTICLE INFO & ABSTRACT \\
\hline Article history: & \multirow{6}{*}{$\begin{array}{l}\text { The aim of this study is to explore the efficacy and safety of metformin in weight loss in Bahraini population. } \\
\text { This study was a cross-sectional survey of } 100 \text { Bahraini patients aged } 20 \text { or more randomly selected. Using a } \\
\text { self-administered questionnaire, volunteers answered questions regarding purposes, doses, side effects and } \\
\text { sources of information of metformin and its impact on weight. Results have shown that } 44 \% \text { of the participants } \\
\text { have experienced weight loss after starting metformin. Weight loss reported was } 1-5 \text { kilograms by } 45.4 \% \text { and up } \\
\text { to } 10 \mathrm{Kg} \text { by } 32 \% \text { of participants. Moreover, } 23 \% \text { of the patients have not experienced any adverse effects after } \\
\text { starting metformin while some patients had abdominal pain }(31 \%) \text { or loss of appetite }(29 \%) \text {.The findings of } \\
\text { this study prove that metformin is an effective drug for weight loss. The results may have been influenced by } \\
\text { other factors such as specific diet and physical exercises .In regard to safety, metformin was found to have a } \\
\text { good safety profile as the side effects reported were mild to moderate and tended to subside after a period of use. }\end{array}$} \\
\hline Received on: 09/02/2016 & \\
\hline Revised on: 18/04/2016 & \\
\hline Accepted on: 23/05/2016 & \\
\hline Available online: $28 / 06 / 2016$ & \\
\hline $\begin{array}{l}\text { Key words: } \\
\text { Metformin, Weight Loss, } \\
\text { slimming, Obesity. }\end{array}$ & \\
\hline
\end{tabular}

\section{INTRODUCTION}

Obesity represents a major factor in the morbidity and mortality of the sufferers and has been described as a risk factor for the development many diseases (Bouza et al., 2012; Lim et al., 2011; Zhang et al., 2014). The obesity control is challenging and includes lifestyle modification in addition to therapeutic approaches that can be followed depending on the severity of the condition (Bouza et al., 2012, Lim et al., 2011). One of the therapeutic approaches that have been described in the literature is the use of biguanides (Bouza et al., 2012; Hasnain et al., 2011; Lim et al., 2011; Malin et al., 2010; Refuerzo et al., 2015). Biguanides are oral hypoglycemic agents used for the management of type 2 diabetes mellitus. Two biguanides; Metformin and phenformin are available for use, although phenformin is not used widely due to high incidence of lactic acidosis associated with its use (Berstein, 2012, Harvey, 2012).

* Corresponding Author

Email: smalqallaf@uob.edu.bh
Biguanides are claimed to reduce blood glucose by reducing hepatic gluconeogenesis, decreasing absorption of intestinal sugar and improving the uptake and utilization of peripheral glucose (Derosa and Maffioli, 2011; Harvey, 2012).Many studies reported weight-reducing effect of metformin in different categories of patients such as patients with diabetes mellitus, polycystic ovarian syndrome, Schizophrenia and obesity (Berstein, 2012; Hasnain et al., 2011; Jarskog et al., 2013; Park et al., 2009; Wang et al., 2012). Effect of metformin on body weight is believed to be due to decrease in food consumption, mainly through direct influence on hypothalamic hormone and might be centrallyvia action on the autonomic nervous system by delaying gastric emptying (Jarskog et al., 2013; Jensterle et al., 2013; Seifarth et al., 2013). However, reducing of insulin resistance and hyperinsulinemia were suggested for weight loss (Berstein, 2012; Zhang et al., 2014). Other actions were also described for the biguanides such as reducing blood lipids, prevention of diabetes and cancer and extension of healthy lifespan (Berstein, 2012; Park et al., 2009; Refuerzo et al., 2015; Zhang et al., 2014). The anti-tumour activity of biguanides and their ability to extend life-span were both linked to their weight reducing effect (Berstein, 2012). 
In regard to metformin safety, studies mentioned that metformin use is associated with several gastro-intestinal adverse effects such as diarrhea, vomiting, flatulence and abdominal discomfort and other effect like headache (Bouza et al., 2012, Cheong, 2013, Refuerzo et al., 2015, Wang et al., 2012,). The aim of this study is to explore the efficacy and safety of metformin in weight loss in Bahraini population. To our knowledge, this is the first study to be done for this aim in Bahrain.

\section{MATERIALS AND METHODS}

A systematic review of the literature using several medical engines including the National Library of Medicine's PubMed database, Science Direct and Google Scholars (limited to English language) was performed. The terms used in the search were metformin, obesity, weight loss, diabetes mellitus, polycystic ovarian syndrome, Metformin adverse effects, metformin for weight loss. The search profile included comprehensive lists of various clinical studies that were conducted in different world areas for statistically relevant information about metformin effect on weight loss. Data of these studies were eventually compared with the analysis of this study, aiming at exploring the efficacy and safety of metformin in weight loss.

A cross sectional survey was carried out to explore the efficacy and safety of metformin in weight loss among the Bahraini population. Using a standardized three paged selfadministered questionnaire, 100 Bahraini patients aged 20 to more than 40 years were interviewed in different private and public outpatient clinics and community pharmacies of different geographical areas in Bahrain. Because of the short deadline for completing the study, convenience sampling was used and therefore the study may not be sufficiently representative of the entire population. The Inclusion criteria were adult diabetic patients attending outpatient clinics and community pharmacies. The exclusion criteria were any patient using any other slimming method was excluded from this study. Both genders were enrolled, in which forty eight males and fifty two females were interviewed. Participants answered several questions regarding the use of metformin, its side effects and sources of their information regarding metformin.

The survey was pilot tested on 10 subjects to improve questions, clarity and quality of data collection. The final survey, however; included 15 questions about different aspects of metformin purposes of usage, frequency and time of administration, awareness of the side effects and sources of information. Full demographic data was collected for all subjects, including age, gender, and weight status (table 1).

A summary score of percent correct answers was finally created. Data were analyzed using the SPSS statistical software Version 20.0. (SPSS Inc. Chicago, IL, USA). Since the study is cross-sectional, descriptive statistical analysis was conducted. This study was approved by the Pharmacy Program Ethics Committees. Data was collected from participants who provided written informed consent.

\section{RESULTS}

Demographical data of participants are shown in Table 1. The female patients were $52 \%$, while the rest were male. The majority $(57 \%)$ of patients were more than 40 years age, $25 \%$ were $30-40$ years and the rest $(18 \%)$ are $20-30$ years. Patients with normal weight were found to be only $42 \%$, while $46 \%$ were overweight and $12 \%$ were obese.

Table 1: Demographical data of participants.

\begin{tabular}{lccc}
\hline \multirow{2}{*}{ Gender } & \multicolumn{2}{c}{ Males } & Females \\
\cline { 2 - 4 } & \multicolumn{2}{c}{$48 \%$} & $52 \%$ \\
\hline \multirow{2}{*}{ Age } & $\mathbf{2 0 - 3 0}$ Years & $\mathbf{3 0 - 4 0}$ Years & $>$ 40 Years \\
& $18 \%$ & $25 \%$ & $57 \%$ \\
\hline \multirow{2}{*}{ Weight status } & Normal & Over weight & Obese \\
& $42 \%$ & $46 \%$ & $12 \%$ \\
\hline
\end{tabular}

DM: Diabetes Mellitus POS: polycystic ovarian syndrome

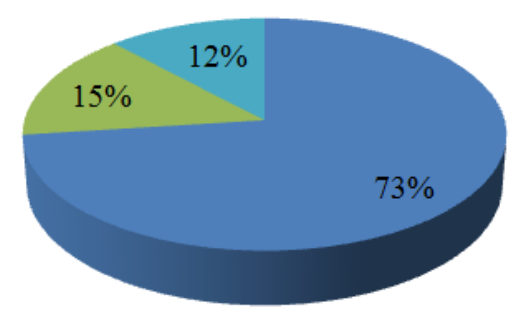

Fig. 1: Purpose of taking Metformin.

Results have shown that $73 \%$ of participants are taking metformin for diabetes mellitus, $15 \%$ for polycystic ovarian syndrome and $12 \%$ of weight loss. The strength of metformin used was found to be $500 \mathrm{mg}$ by $52 \%$ of patients, and $1000 \mathrm{mg}$ by $47 \%$ of patients(Figure 2A) and used most commonly (54\%) at a twice daily dosage regimen (Figure 2B).
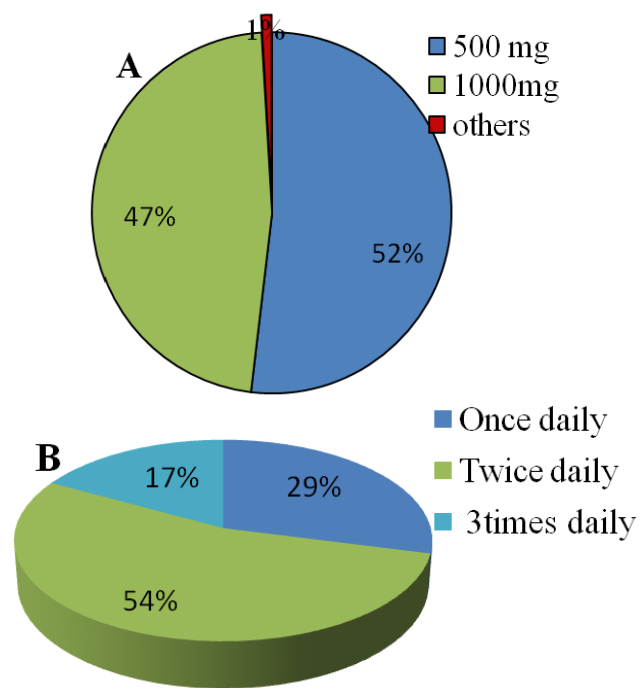

Fig. 2: Metformin Strength used (A) and dosage regimen (B).

The Results revealed that the majority (95\%) of patients has started metformin following doctors' prescription, but a minority of them have heard about it from pharmacists, 
television, social networks (Instagram) or friends and relatives (Figure 3). The intake of metformin in regard to food was found to be correct in $66 \%$ of patients, but a good number of patients are using it before food or anytime (Figure 4).

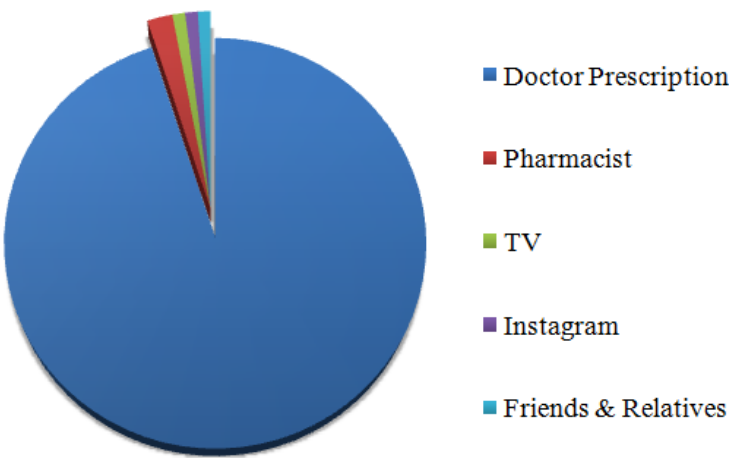

Fig. 3: Sources of first knowledge of Metformin.

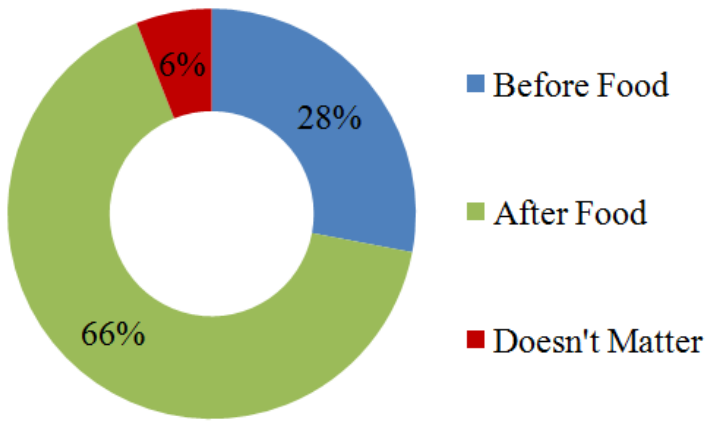

Fig. 4: Taking Metformin in regard to food.

Most patients have been using metformin since more than a year (53\%), while the rest of them have been using it since less than a year (Figure 5).

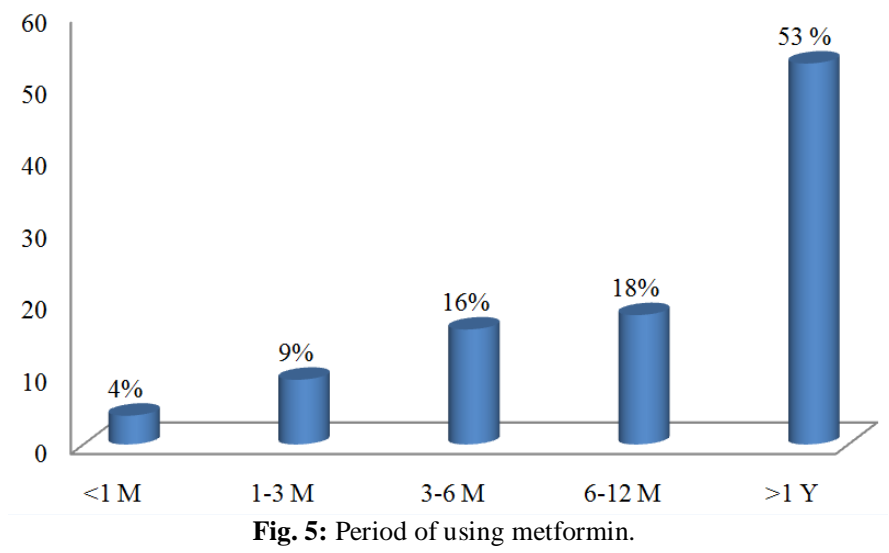

Moreover, $23 \%$ of the patients have not experienced any adverse effects after starting metformin while some patients had abdominal pain $(31 \%)$, loss of appetite $(29 \%)$, and minorities had nausea, vomiting, diarrhea and other side effects such as sweating, flatulence, constipation, and dizziness (Figure 6).

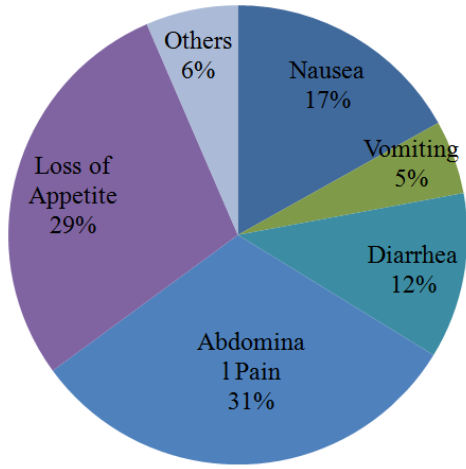

Fig. 6: Adverse effects experienced by patients after starting metformin

Interestingly, a significant number $(44 \%)$ of patients has reported weight loss after starting metformin, but the rest has not experienced any weight loss (Figure7). Out of these, $45.4 \%$ of participants had a weight loss of 1 to $5 \mathrm{Kg}$ and weight loss of up to $10 \mathrm{Kg}$ was reported by approximately $32 \%$ of participants (Figure $8)$.

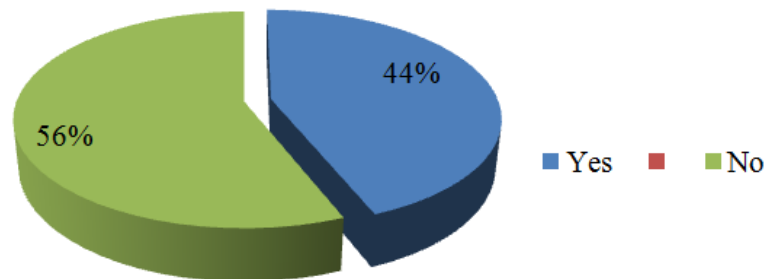

Fig. 7: Weight loss after starting metformin.

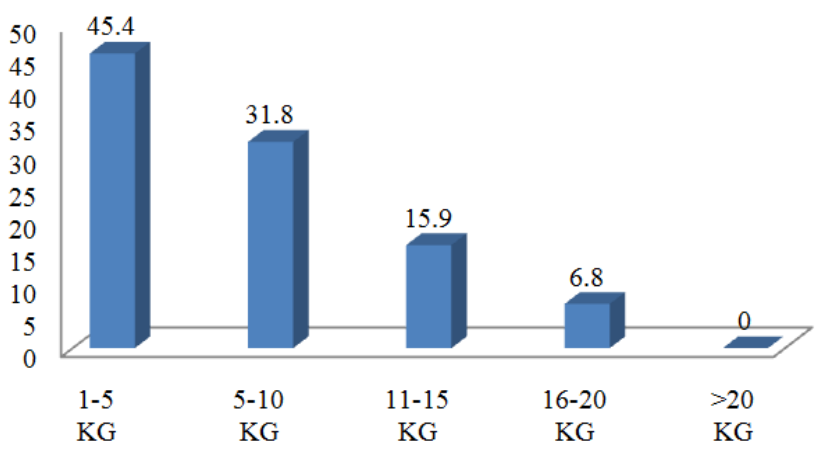

Fig. 8: Number of kilograms lost after starting metformin.

Weight loss that occurred after starting metformin was believed to be caused by the drug in $48 \%$ of the participants (Figure 9).

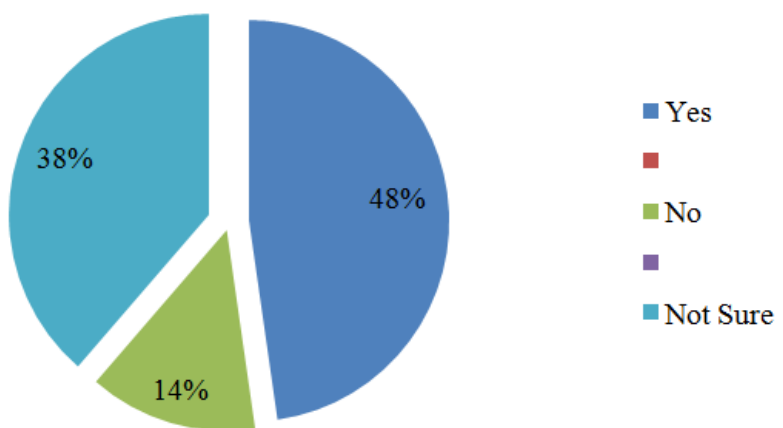

Fig. 9: Patients belief that weight loss was caused by metformin. 
A significant number of patients were found to follow a specific diet and physical exercises even before starting metformin (Figures 10 and 11).
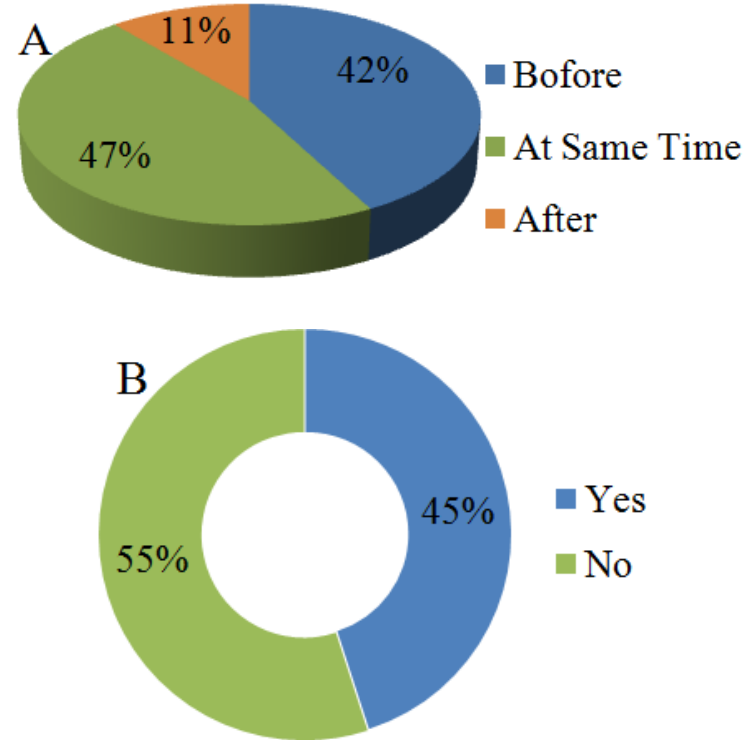

Figure 10: Patients following specific diet (A) and time of starting this diet (B).
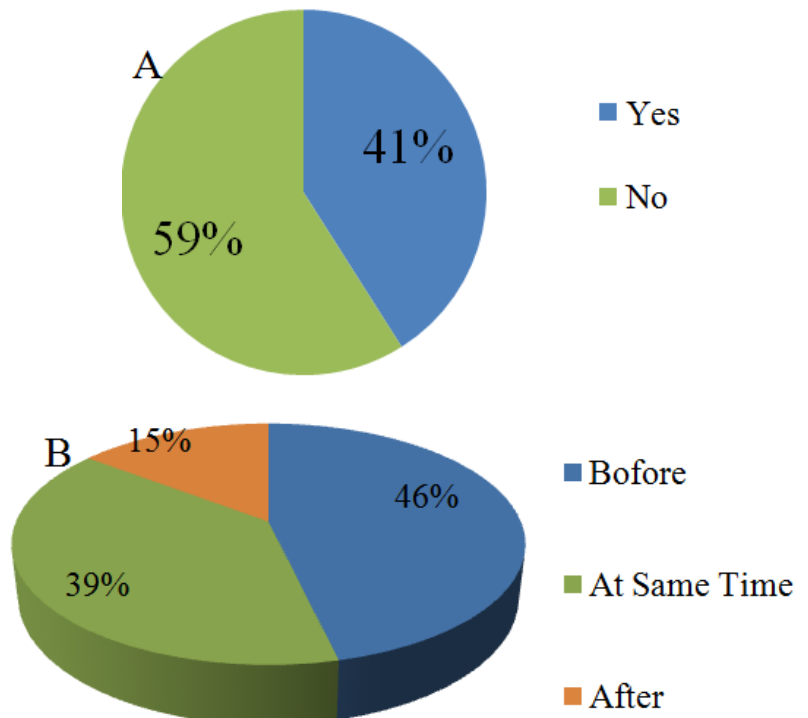

Fig. 11: Patients doing physical exercises (A) and time of starting these exercises (B).

\section{DISCUSSION}

This study represents an attempt to explore the efficacy and safety of metformin on weight loss among the Bahraini population and their knowledge in this regard. The results showed that both men and women are using metformin at almost the same percentage. The most common purpose of the use was diabetes mellitus, although poly-cystic ovarian syndrome and weight loss were also among the purposes of use. The number of participants that are self-medicating with metformin was found to be nonsignificant as the majority of patients are using it further to physicians' prescriptions. The doses used were 500mg $-1000 \mathrm{mg}$, once or twice daily with a few cases of 3 times daily with the majority of patients using it for more than one year. The use of metformin in regard to food was found to be correct in the majority of patients reflecting good compliance. The side effects experienced by the patients further to the metformin use were found to be mild to moderate and are similar to those reported in the literature with the abdominal pain and loss of appetite being the highest. These side effects tend to subside with the continuous use of the drug as reported by the literature and the patients in this study; although some patients mentioned that they were not sure if the drug was the cause of these side effects. Significant number of patients believes that metformin caused them weight loss that reached up to 15 kilograms or more since they started its use. This weight loss is most probably caused by metformin itself as the majority of patients mentioned that they haven't followed any lifestyle modification during the drug treatment. However, the influence of life-style modification could not be denied as a significant number of patients mentioned following special diet and doing physical exercises along with metformin use.

The current study is in accordance with the findings of previous studies that have shown metformin efficacy and safety in body weight reduction (Al-nozha et al., 2013, Berstein 2012, Bouza et al., 2012; Jarskog et al., 2013; Park et al., 2009; Refuerzo et al., 2012; Seifarth et al., 2013; Wang et al., 2012). The efficacy of metformin in weight loss was established in different categories of patients, including diabetes mellitus, polycystic ovarian syndrome, obesity and patients treated with antipsychotic drugs. Many studies discussed the use of metformin for weight loss in women with poly-cystic ovarian syndrome and addressed its efficacy. The study of Al-Nozha 2013 found that metformin combined with lifestyle modification (diet restriction or exercise) resulted in a significant weight loss of an average $5.3 \mathrm{~kg}$ in 6 months in patients with poly-cystic ovarian syndrome (AlNozha et al., 2013). Other studies expressed the weight loss secondary to metformin use as percentage rather than the number of kilograms lost and indicated its long-term efficacy and safety (Berstein 2012; Wang et al., 2012; The diabetes prevention program research group, 2012).

The Combination of metformin and lifestyle changes was proved to be superior to either approach alone. Metformin in combination with structured lifestyle modification programs was capable of achieving modest reduction in BMI that was significantly higher than that achieved with lifestyle modification alone. This effect was reported in a study on patients that were regarded to be refractory to lifestyle modification (Bouza et al., 2012). Although, some studies reported that comprehensive lifestyle modification induced higher weight loss compared to metformin (Lim et al., 2011), other studies have proved that combining both approaches would produce better results than single approach alone (Al-nozha et al., 2013; Bouza et al., 2012; Malin et al., 2010).

Combination of metformin with some other anti-diabetic agents was found to be superior to metformin alone. Example of these anti-diabetic agents is Glucagon-like peptide-1 (GLP1) 
analog; liraglutide (Jensterle et al., 2013) and the thiazolidindiones (Derosa and Maffioli, 2011). Previous studies have shown that there was no statistical difference in regard to safety profile or withdrawal between metformin and placebo (Bouza et al., 2012; Jarskog et al., 2013; Refuerzo et al., 2012; The diabetes prevention program research group, 2012).

In conclusion, the combination of metformin with lifestyle modification represents a potentially effective and safe approach for the control of obesity. Further studies are needed to prove the long-term safety and efficacy of this approach. Small number of participants and the impact of lifestyle habits on metformin induced weight loss need to be taken into consideration. Adjusting weight loss to actual patients' weight and the exact period of use was not possible due to the scope of the current study and the time frame.

Conflict of Interest statement: None

Acknowledgment: Author is gratefully acknowledge Ms. Azhar Jaffar for her assistance in conducting this study.

\section{REFERENCES}

AL-Nozha O, Habib F, Mojaddidi M, Fath El-Bab M Body weight reduction and metformin: Roles in polycystic ovary syndrome. Pathophysiology, 2013; 20: 131-137.AG

Berstein LMMetformin in obesity, cancer and aging: addressing controversies. Aging,2012; 4 (5); 320-329.

Bouza C, López-Cuadrado T, Gutierrez-Torres LF, Amate J. Efficacy and Safety of Metformin for Treatment of Overweight and Obesity in Adolescents: An Updated Systematic Review and MetaAnalysis. Obesity Facts, 2012; 5:753-765.

Cheong B. Metformin: A Differential Diagnosis for Weight Loss, Altered Bowel Habits and B12 Deficiency Anemia in an Elderly Diabetic. Medical Journal of Malaysia,2013; 68 (5): 441-442.

Derosa G, Maffioli P. Thiazolidinediones plus metformin association on body weight in patients with type 2 diabetes. Diabetes Research and Clinical Practice,2011; 91: 265-270.

Harvey, RA. 2012. Lippincott's Illustrated Reviews: Pharmacology. $5^{\text {th }}$ ed. Philadelphia: Lippincott Williams \&Wilkins.

Hasnain M, Fredrickson SK, Victor W, Vieweg R. Metformin for obesity and glucose dysregulation in patients with schizophrenia receiving antipsychotic drugs. Journal of Psychopharmacology, 2011; 25(6): 715-721.
Jarskog LF, Hamer RM, Catellier DJ, Stewart DD, LaVange L, RayN, et al.. Metformin for weight loss and metabolic control in overweight outpatients with schizophrenia and schizoaffective disorder, The American Journal of Psychiatry, 2013; 170 (9): 1032-1040.

Jensterle Sever M, Kocjan T, Pfeifer M, Kravos NA and Janez A. Short-term combined treatment with liraglutide and metformin leads to significant weight loss in obese women with polycystic ovary syndrome and previous poor response to metformin. European Journal of Endocrinology,2013;170 (3): 451-459.

Lim SS, Norman RJ, Clifton PM, Noakes M.The effect of comprehensive lifestyle intervention or metformin on obesity in young women. Nutrition, Metabolism \& Cardiovascular Diseases, 2011; 21: 261268.

Malin S.K., Choi S., Thistle L.J., Braun B. Effects of Exercise Training and/or Metformin for 12 weeks on Body Weight and Energy Intake in Individuals with Prediabetes. Journal of the Academy of Nutrition and dietetics, 2010; 110 (9); Supplement A40.

Park MH, Kinra S, Ward KJ, White B, Viner RM. Metformin for Obesity in Children and Adolescents: A Systematic Review. Diabetes Care, 2009; 32 ( 9): 1743-1745

Refuerzo JS, Viteri OA, Hutchinson M, Pedroza C, Blackwell $\mathrm{SC}$, Tyson JE, et al. . The effects of metformin on weight loss in women with gestational diabetes: a pilot randomized, placebo-controlled trial. American Journal of Obstetrics and Gynecology, 2015; 212:389.e1-9.

Seifarth C, Schehler B, Schneider HJ. Effectiveness of Metformin on Weight Loss in Non-Diabetic Individuals with Obesity. Expert Clinical Endocrinology Diabetes, 2013; 121: 27-31.

The Diabetes Prevention Program Research Group. Long-Term Safety, Tolerability, and Weight Loss Associated With Metformin in the Diabetes Prevention Program Outcomes Study. Diabetes Care,2012; 35: 731-737.

Wang M, Tong J, Zhu G, Liang G, Yan H, Wang X. Metformin for treatment of antipsychotic-induced weight gain: A randomized, placebo-controlled study. Schizophrenia Research, 2012; 138:54-57.

Zhang Q, Schmandt R, Celestino J, McCampbell A, Yates MS, Urbauer DL, et al. CGRRF1 as a novel biomarker of tissue response to metformin in the context of obesity. Gynecologic Oncology, 2014; 133:83-89

\section{How to cite this article:}

Sayed Mahmood Al-qallaf. Efficacy and Safety of Metformin In Weight Loss in Bahraini Population. J App Pharm Sci, 2016; 6 (06): 091-095. 